\title{
Research on Human Skin Condition Evaluation based on Neural Network
}

\author{
Chang Liu ${ }^{1, a}$, Li Wang ${ }^{2, b}$, Xiaoyi Wang ${ }^{2, c}$, Jiping $\mathrm{Xu}^{2, \mathrm{~d}}$,Huiyan Zhang, ${ }^{2, \mathrm{e}}$, \\ Yinmou Dong ${ }^{2, f}$ \\ ${ }^{1}$ Center of China Cosmetic Research, Beijing Technology and Business University, Guangzhou \\ 510000, China; \\ a1553928775@qq.com, bbtbu_wl@126.com
}

Keywords: neural network, classification, the status of human skin, evaluation.

\begin{abstract}
With the continuous development of peoples' life, the skin-care is attracting more attention. There are so many factors affecting the skin condition, and there is nonlinear correlation between factors. However, the current research always use single index to evaluate skin condition which would not be accurate. This article is to introduce seven key factors which affect the skin conditions into the evaluation. The skin condition classification model is built by the BP neural network according to the skin condition index. Firstly, on the basis of age, the skin condition is classified into three type: youth, middle aged, and the old, then the feature of each kind is extracted, and then according to the seven key factors, the neural network calculation is used to study the skin condition, finally, the neural network classification result is compared with the real age to complete the evaluation. The result proves that, by using the method this article introduced in the classification of skin condition, the similarity with the real age can be 70\%, BP neural network can evaluate the skin condition effectively. In addition, the method is simple and practical which supplies effective way to do the evaluation. The physical significance of the evaluation is clear and it uses the difference-information in maximum which is the accurate and effective way to obtain the skin condition information.
\end{abstract}

\section{Introduction}

With the enhancement of people's health awareness, people pay more and more attention to the health and beauty of skin. Especially women begin to care about how to improve it. In order to establish a unified and reasonable evaluation criterion of human skin, it is needed to use more accurate method to evaluate the skin of human body more objectively.

At present, some scholars at home and abroad are working on skin evaluation, Chen Jin and others have studied in the quantitative evaluation of roughness of facial skin and its application in medical cosmetology field, and analyzed the importance of human skin texture in the beauty and nursing care[1];Wen Xiang et al. Evaluated the relationship between age and skin texture, roughness and elasticity by studying three kinds of non - invasive methods, which can provide reference for the determination of skin evaluation index[2].Zhang Mi et al. Established a unified system about skin image acquisition, evaluation and management, based on evaluating the skin image feature parameters of the 24 year old woman, and it can accurately reflect the current state of skin[3];Xu Pengxiang proposed the use of the index value of the image to evaluate the quality of skin, the study uses neural network to make evaluation of skin aging, which has a certain guiding significance[4];Gu Chunjing et al. [5] introduced the development of the technique of skin surface detection and parameter analysis at home and abroad, and summarized various methods for the evaluation of skin texture. Hang, Yong-Tai et al. [6] studied the affected factors of the skin vitality, and investigated the effect of skin vitality, alcohol liposome and liposome in the evaluation of skin deposition; Tang Li et al. [7] proposed the evaluation of skin elasticity, and the study of skin elasticity is quantified, so that the evaluation of the skin elasticity has an objective criteria. In foreign countries, in recent years, with the development of high and new technology, using laser scanning or confocal microscopy and 
computer image analysis and recognition technology for skin surface structure research, the skin surface structure can be fine analysis and reproduce the three-dimensional structure of the skin.

However, the skin evaluation system is not very perfect in our country. The measurement of the parameters about human skin is single on the existing domestic market. limited by the level of hardware technology, a skin measuring instrument can only measure $1 \sim 3$ parameters, including oil, moisture and elasticity. What's more those various parameters are independent of each other, and there are no combined analysis of the relationship among them. The oil content measurement mainly adopted optical type; Moisture measuring electrostatic capacity type; Elastic measurement of ultrasonic vibrating. In addition, there are no details about error and precision. Without the measurement results being quantitative indicators, it is hard to make a transverse comparison. All key indicators which affect the the state of the skin can not to be applied in most evaluation system. Most scholars only consider the single index in their study. So that their result not only is with larger error but also cannot fully reflect the state of the skin. Some other scholars evaluate the state of the skin by processing the image to extract target. But this method need the high requirement of expensive equipment.

Therefore, in this paper, based on the statistical process of the most index that affect state of the human skin, seven key indicators which are acquired by the BP neural network algorithm are integrated to reflect differences in human skin information ,from the greatest extent and with minimum indicators. We obtain the owner's age range by analysis of their skin condition, then compare the results with the owner's real age to text whether the measured skin is in line with the condition it should be.

\section{The experimental scheme and skin status indicators}

\subsection{Section Headings.}

To research the method of skin condition evaluation which is proposed in this paper, collaborative innovation center of Beijing technology and business university do experiments in human skin condition evaluation, so that the data of skin status evaluation can be collected, for researching the method in this paper and verifying the experiments. There are 853 groups of human skin samples collected in the experience. The age of those people who join the collection is from 16 to 64, and the sex ratio is 1:3. The 853 groups of experimental data samples, according to the age, can be divided into three categories, which are youth age, middle age and old age. In the process of using neural network to evaluate the skin, the "1"," 2 ", “3”, are respectively on the output of the three age groups. Furthermore, 17-24 is young age ,25 to 49 is middle age and 50 to 64 is old age.

To eliminate the influence of different skin condition of body parts, each kind of the indicators associated with human skin state would collect seven parts of body as the experimental data including left forehead, left cheek, chin, Adam's apple, left arm, left hand, and right hand side, and then average seven parts of the experimental data. Because there are so many types associated with human skin condition index, if each indicators are disposed, it will cause too much processing data and unnecessary error processing results, and more difficult to process. What's more, found by observing the experimental data, some indicators have a linear correlation including the hemoglobin and melanin, the average roughness $\mathrm{Rz}$, smooth skin depth $\mathrm{Rp}$, arithmetic average roughness $\mathrm{Ra}$, and the values of $\mathrm{L}$, a, b value. After averaging the above indexes, three kinds of indicators including the red melanin mean, average roughness, skin Lab values are got. And coupled with other indicators, the seven key indicators associated with human skin state are obtained, namely the average moisture content and moisture loss mean average, $\mathrm{PH}$ value, grease mean, red average melanin, skin LAB values, and the average roughness of the skin. As shown in table 1 
Table 1 Index number

\begin{tabular}{|c|c|c|c|c|c|c|c|c|c|c|c|c|}
\hline & $\begin{array}{c}\text { Water } \\
\text { content }\end{array}$ & $\begin{array}{c}\text { Water } \\
\text { lost }\end{array}$ & $\mathrm{PH}$ & $\begin{array}{l}\text { Oil and } \\
\text { grease }\end{array}$ & $\begin{array}{c}\text { Red } \\
\text { pigment }\end{array}$ & $\begin{array}{c}\text { Black } \\
\text { pigment }\end{array}$ & $\mathrm{L}$ & A & B & $\mathrm{Rz}$ & $\mathrm{Rt}$ & $\mathrm{Rm}$ \\
\hline $\begin{array}{c}\text { Forehea } \\
\text { d }\end{array}$ & $\mathrm{a}_{11}$ & $a_{12}$ & $a_{13}$ & $a_{14}$ & $\mathrm{a}_{15}$ & $\mathrm{a}_{16}$ & $\mathrm{a}_{17}$ & $\begin{array}{c}\mathrm{a}_{1} \\
8\end{array}$ & $\mathrm{a}_{19}$ & $\mathrm{a}_{110}$ & $a_{111}$ & $\mathrm{a}_{112}$ \\
\hline Chin & $a_{21}$ & $a_{22}$ & $a_{23}$ & $a_{24}$ & $a_{25}$ & $\mathrm{a}_{26}$ & $a_{27}$ & $\begin{array}{c}\mathrm{a}_{2} \\
8\end{array}$ & $a_{29}$ & $a_{210}$ & $a_{211}$ & $\mathrm{a}_{212}$ \\
\hline $\begin{array}{c}\text { Left } \\
\text { cheek }\end{array}$ & $a_{31}$ & $a_{32}$ & $a_{33}$ & $a_{34}$ & $a_{35}$ & $a_{36}$ & $a_{37}$ & $\begin{array}{c}a_{3} \\
8\end{array}$ & $a_{39}$ & $a_{310}$ & $a_{311}$ & $a_{312}$ \\
\hline $\begin{array}{c}\text { Adam's } \\
\text { apple }\end{array}$ & $\mathrm{a}_{41}$ & $a_{42}$ & $a_{43}$ & $a_{44}$ & $\mathrm{a}_{45}$ & $\mathrm{a}_{46}$ & $\mathrm{a}_{47}$ & $\begin{array}{c}\mathrm{a}_{4} \\
8 \\
\end{array}$ & $\mathrm{a}_{49}$ & $a_{410}$ & $a_{411}$ & $\mathrm{a}_{412}$ \\
\hline Left arm & $a_{51}$ & $a_{52}$ & $a_{53}$ & $a_{54}$ & $a_{55}$ & $a_{56}$ & $a_{57}$ & $\begin{array}{c}\mathrm{a}_{5} \\
8\end{array}$ & $a_{59}$ & $a_{510}$ & $a_{511}$ & $a_{512}$ \\
\hline $\begin{array}{c}\text { The left } \\
\text { hand } \\
\text { side }\end{array}$ & $a_{61}$ & $a_{62}$ & $a_{63}$ & $a_{64}$ & $a_{65}$ & $a_{66}$ & $a_{67}$ & $\begin{array}{c}\mathrm{a}_{6} \\
8\end{array}$ & $a_{69}$ & $a_{610}$ & $a_{611}$ & $a_{612}$ \\
\hline $\begin{array}{c}\text { The } \\
\text { right } \\
\text { hand } \\
\text { side }\end{array}$ & $a_{71}$ & $a_{72}$ & $a_{73}$ & $a_{74}$ & $a_{75}$ & $a_{76}$ & $a_{77}$ & $\begin{array}{c}a_{7} \\
8\end{array}$ & $a_{79}$ & $a_{710}$ & $a_{711}$ & $a_{712}$ \\
\hline
\end{tabular}

Seven types of index are calculated as formula (1) - (7)

The average moisture content $=\frac{\sum_{i=1}^{7} a_{i 1}}{7}$

Average moisture loss $=\frac{\sum_{i=2}^{7} a_{i 2}}{7}$

The average $\mathrm{PH}=\frac{\sum_{i=3}^{7} a_{i 3}}{7}$

The average oil $=\frac{\sum_{i=4}^{7} a_{i 4}}{7}$

Average red pigment melanin $=\frac{\sum_{i=5}^{7} a_{i 5}+\sum_{i=6}^{7} a_{i 6}}{14}$

The average LAB value $=\frac{\sum_{i=7}^{7} a_{i 7}+\sum_{i=8}^{7} a_{i 8}+\sum_{i=9}^{7} a_{i 9}}{21}$

The average roughness $=\frac{\sum_{i=10}^{7} a_{i 10}+\sum_{i=11}^{7} a_{i 11}+\sum_{i=12}^{7} a_{i 12}}{21}$

Figure 1-- 14is the average of the seven indexes under each age.And In order to be able to understand the relationship more clearly between the experimental data, In this paper, the raw data plotted in Fig. The figure can be clearly seen the distribution in the various types of indicators youth, middle age, elderly. Figure 8 - 14 are seven indexes of the original data. 


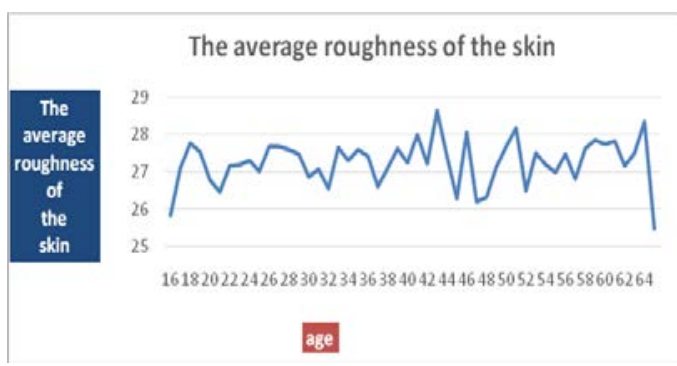

Fig. 1 The average roughness of the skin

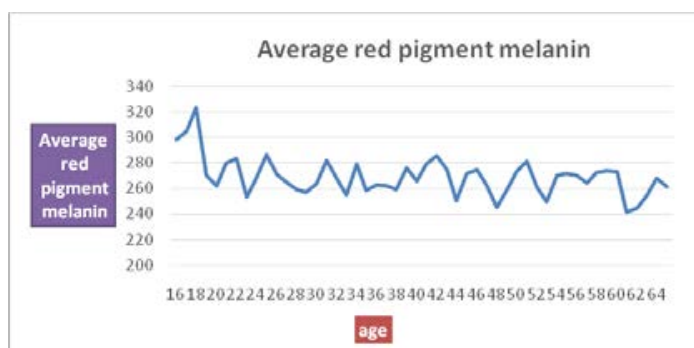

Fig. 3 Average red pigment melanin

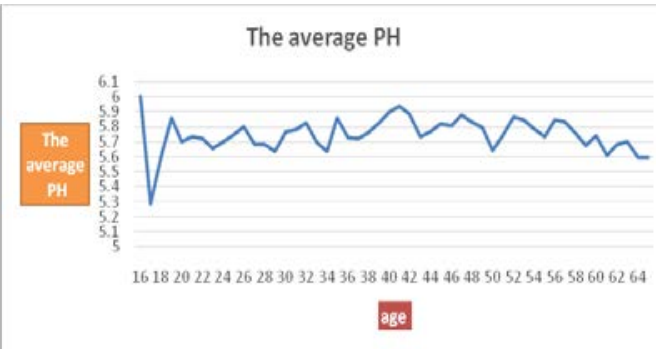

Fig. 5 The average $\mathrm{PH}$

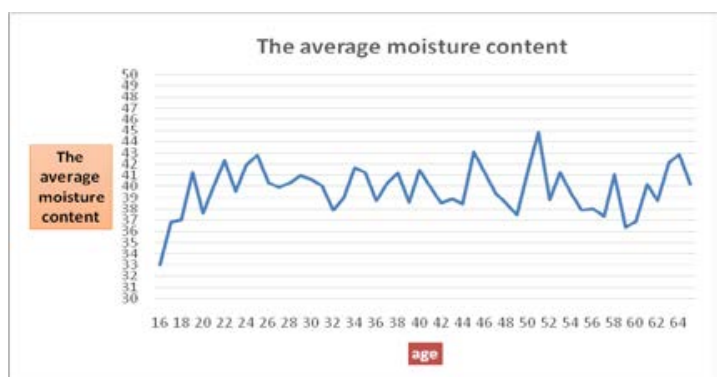

Fig. 7 The average moisture content

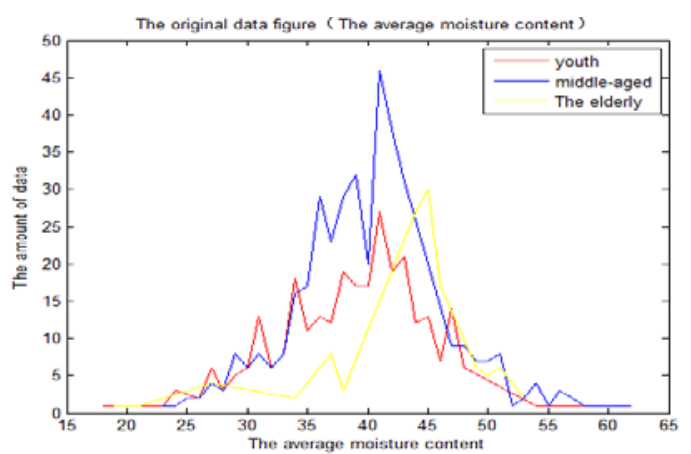

Fig. 9 Average moisture content of the original data data

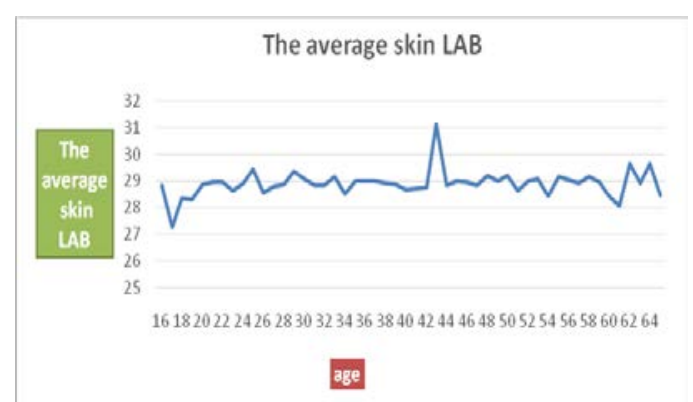

Fig. 2 The average skin LAB

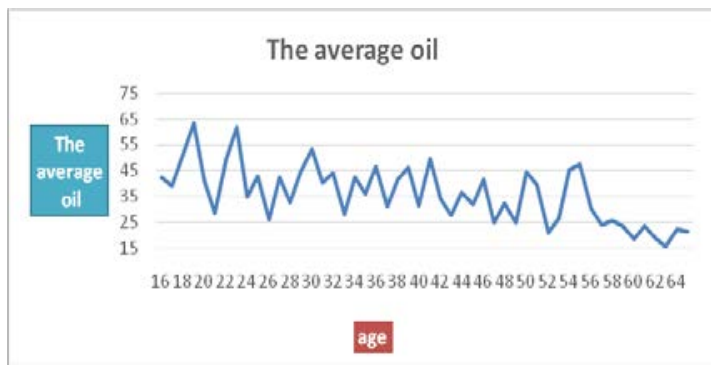

Fig. 4 The average oil

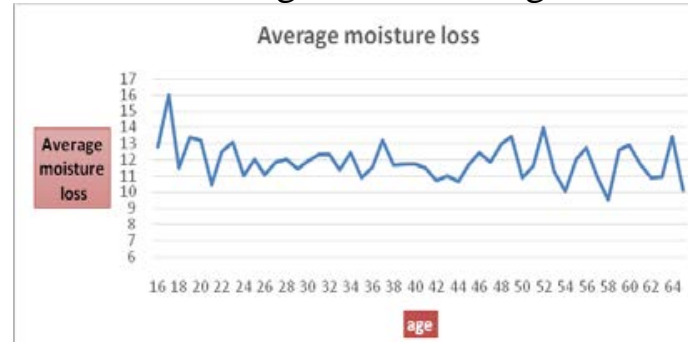

Fig. 6 Average moisture loss

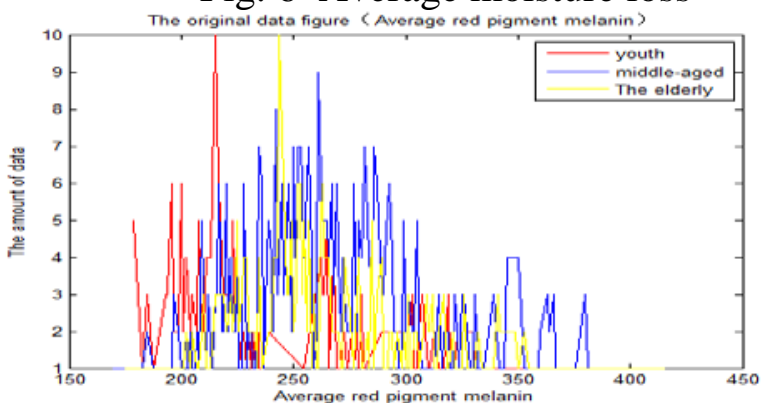

Fig. 8 Average red pigment melanin of the skin the original

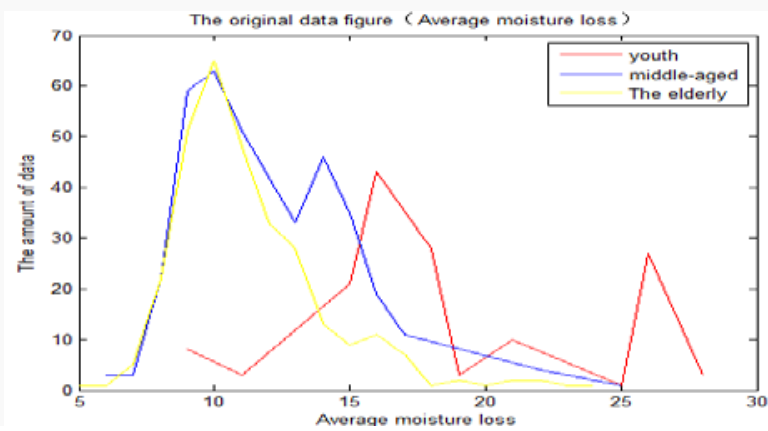

Fig. 10 Average moisture loss of the original data 


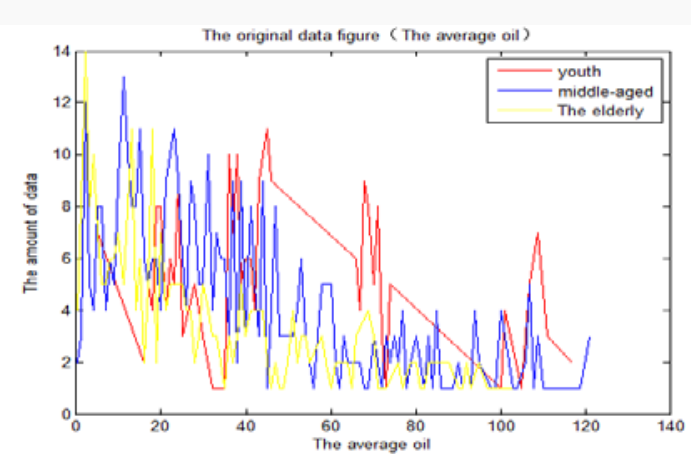

Fig. 11 The average oil of the original data

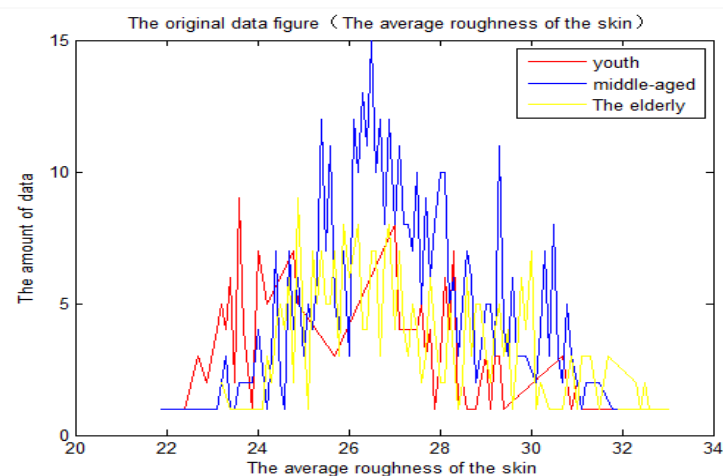

Fig. 13 The average roughness of the skin the original data

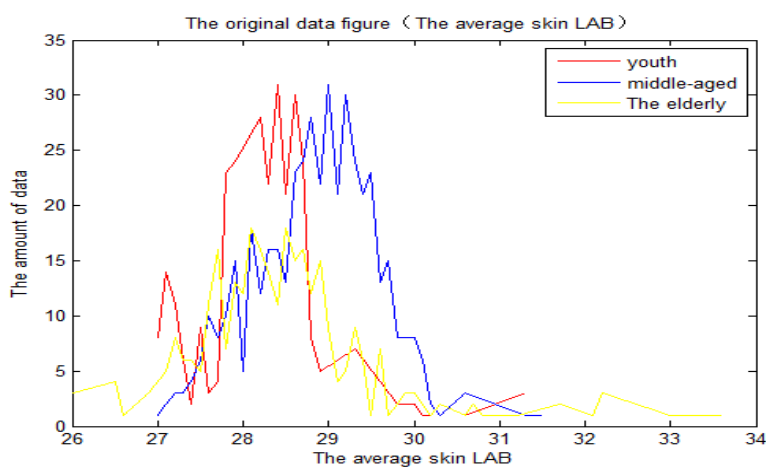

Fig. 12 The average skin LAB the original data

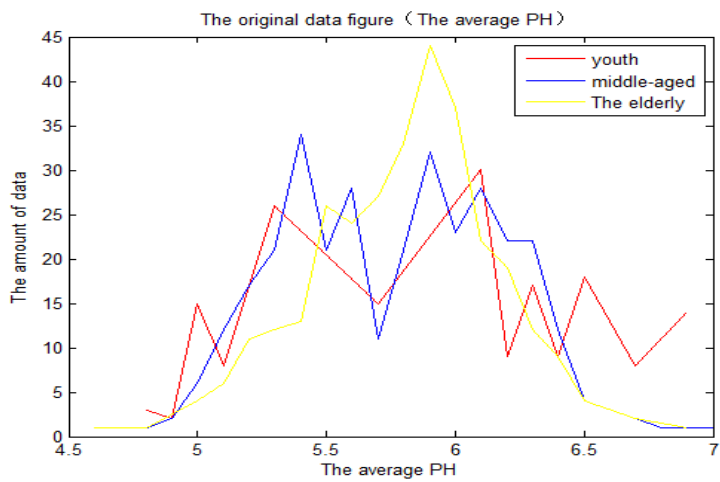

Fig. 14 The average $\mathrm{PH}$ of the skin the original data

According to the figure 9-15, any single indicator is not significantly distinguish the differentce about the state of human body skin, namely the classification of the age.to the greatest extent reflect the differences of human skin state information, this paper comprehensive used seven indexes to characterize the features of all kinds of skin condition.

\section{The classification of human skin condition based on neural network modeling}

Classifying the skin of human is a complex not-liner problem. Because the BP artificial neural network has the powerful ability of disposing not-liner system [8], and of identifying and classifying outdoor input samples, and it can classify the not-liners better. As a result, this paper uses the BP artificial neural network to study the classification problem of the state of human skin, founding BP network model skin of condition classification.

BP neural network classification's performance depends on the training samples, which means choosing data from the existing sample selection in order to get high quality of the training so that effectively improve the performance of the neural network classifier. Seeming 853 collected in the experiment were seven indexes as the classified samples, choosing selected from 750 samples as training samples randomly, the remaining 103 samples will be identified samples. Part of the experiment data are shown in table 2 . 
Table 2 sample data

\begin{tabular}{ccccccccc}
\hline $\begin{array}{c}\text { Sample } \\
\text { number }\end{array}$ & Age & $\begin{array}{c}\text { Average } \\
\text { moisture } \\
\text { content }\end{array}$ & $\begin{array}{c}\text { Average } \\
\text { moisture } \\
\text { loss }\end{array}$ & $\begin{array}{c}\text { Average } \\
\text { PH }\end{array}$ & $\begin{array}{c}\text { Average } \\
\text { oil }\end{array}$ & $\begin{array}{c}\text { Average red } \\
\text { pigment } \\
\text { melanin }\end{array}$ & LAB & $\begin{array}{c}\text { Average } \\
\text { roughness }\end{array}$ \\
\hline 1 & 3 & 34.44 & 11.81 & 6.12 & 75.66 & 231.00 & 28.11 & 24.55 \\
\hline 2 & 2 & 55.71 & 10.74 & 5.78 & 21.33 & 228.00 & 28.98 & 26.71 \\
\hline 3 & 3 & 39.57 & 17.15 & 6.00 & 3.00 & 238.42 & 28.67 & 27.47 \\
\hline 4 & 3 & 39.50 & 12.86 & 5.77 & 19.66 & 279.14 & 29.33 & 25.19 \\
\hline 5 & 3 & 50.56 & 15.80 & 5.97 & 43.66 & 270.78 & 29.37 & 25.70 \\
\hline 6 & 3 & 40.81 & 13.75 & 4.74 & 18.33 & 268.00 & 29.17 & 25.17 \\
\hline 7 & 3 & 36.37 & 16.61 & 5.34 & 2.00 & 249.07 & 29.13 & 25.58 \\
\hline 8 & 3 & 38.11 & 13.32 & 5.61 & 27.33 & 261.50 & 29.18 & 26.37 \\
\hline 9 & 2 & 38.03 & 17.51 & 5.94 & 61.00 & 264.64 & 29.03 & 25.74 \\
\hline 10 & 2 & 42.16 & 28.68 & 5.90 & 87.66 & 311.21 & 28.22 & 25.53 \\
\hline 11 & 3 & 37.54 & 17.28 & 5.52 & 18.33 & 251.64 & 28.76 & 24.78 \\
\hline 12 & 2 & 28.41 & 11.14 & 5.40 & 94.33 & 257.21 & 28.61 & 25.58 \\
\hline 13 & 2 & 28.36 & 13.08 & 5.92 & 78.33 & 264.50 & 28.05 & 27.29 \\
\hline 14 & 2 & 35.16 & 10.14 & 5.97 & 98.00 & 219.42 & 28.57 & 25.42 \\
\hline 15 & 3 & 38.64 & 15.88 & 5.47 & 3.00 & 236.14 & 29.20 & 25.85 \\
\hline
\end{tabular}

Due to the differences in index values for each test subject of experiment is too large,in order to prevent the large numerical information annexation of small numerical information, normalized Index values for all tested. Using the formula (8) which ranges all normalized to 0-1.

$$
X_{i}=\frac{x_{i}-x_{\min }}{X_{\max }-x_{\min }}
$$

Among them, $x_{i}$ for each index of the I sample data, $x_{\min }, x_{\max }$ for the index of all sample data for the minimum and maximum values, after formula (1), all the values are in $[0,1]$.

Three layer of the skin of BP network classification model is constructed and its structure model as shown in Figure 15: 7 input nodes, $x_{1}, x_{2}, x_{3}, x_{4}, x_{5}, x_{6}, x_{7}$ respectively, on behalf of the rough skin, average value, average value of LAB, red pigment average value, average value of oil, average value of $\mathrm{pH}$, water loss average value, average values of moisture content; $\mathrm{y}_{1}, y_{2}, \ldots, y_{n}$ hidden layer nodes; three output nodes number: 1, 2, 3; 1 represents the output for young age, 2 represents the output for middle-aged age, three representative output for old age.

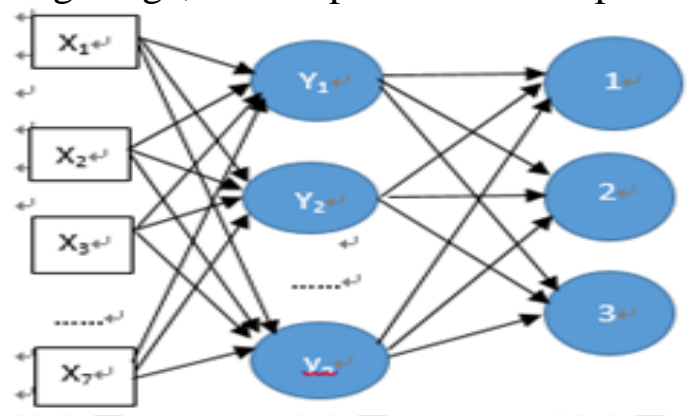

The input layer Hidden layer Output layer

Fig. 15 Three layers BP network model structure

In the selection of the hidden layer node number, when the node number is too small, each node is overloaded, the number of iterations is high, the pattern classification identification rate is reduced. When the node number is large, the burden of each node will be reduced, the pattern recognition rate is improved, but the computation amount will be significantly increased. Therefore, the implicit layer node number is determined by the empirical formula of the single hidden layer node:

$n 1=n+0.618(n-m)$

(6)

Among them, $\mathrm{n}$ is the number of input nodes, $\mathrm{m}$ is the number of output nodes. 
In the aspects of the quantitative detection method of the quality of human skin, the documents suggest that the skin elasticity and the $\mathrm{PH}$ are index of human skin conditions that varies(changes) greatly obvious as the age changes. That researches show that the $\mathrm{PH}$ ranges from 5.3 to 5.5 in young period. During youth period the value of $\mathrm{PH}$ is 5.5 to 5.8, while during the middle age the value of $\mathrm{PH}$ is 5.4 to 5.7. During the Juvenile period the value of the skin elasticity is 0.57 to 0.59 . Also during the youth period the value of the elasticity is 0.52 to 0.57 . And during the middle age the value of the skin elasticity is 0.48 to 0.52 . We evaluated the facial skin conditions according to two indexes which are the value of the $\mathrm{PH}$ and elasticity. The correct rate of the result is $43.29 \%$ by this standard.

This article uses the BP neural network to classify and evaluate the human skin conditions.

When the neural network hidden nodes is 6 , the correct rate of skin condition classification is the highest. The value of the correct rate is $70 \%$. This skin condition classification model achieves a certain accuracy. The chart 16 shows that the classification of 103 Identification samples during the current age and the tested age.

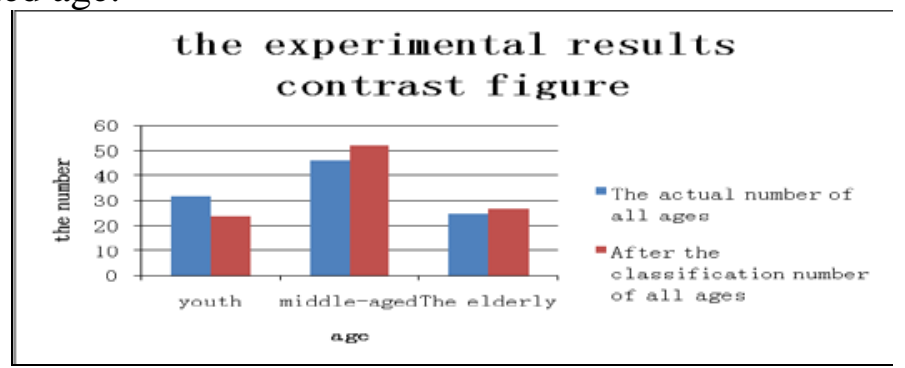

Fig. 16 Experimental results contrast figure

Through examples proved that when the training data sample is large enough, this article proposed which based on the seven indexes of BP artificial neural network classification method can ensure the accuracy of the results by the human body skin, make the method has practical significance.

\section{Conclusion}

In this paper, the human body skin state are classified according to the age. A new method of classification assessment of the state of skin is established in terms of the index of the seven key human skin class, by the BP artificial neural network based on the technology of artificial intelligence theory,. The method founds the potential contact between the indicators of skin condition and age, estimates the state of the human body skin ages according to the state of human body skin indicators, and judges whether the state of facial skin age conform to the actual state of facial skin ages age by compared with actual age. In addition, it provides objective and reliable reference basis for improving the skin condition, and used for the diagnosis of skin and beauty products research and guidance of development.

\section{Ackowlegements}

This work financially supported by the comprehensive reform of personnel training project of Beijing Technology and Business University----Research Center of Chinese cosmetic collaborative innovation under Grant No. 19008001060.Those supports are gratefully acknowledged.

\section{References}

[1]. Jin Chen. The quantitative evaluation of facial skin roughness and its application in medical beauty [D]. University of electronic science and technology, 2009.

[2]. Wen Xiang Jiang Xian, BianCaiYun, etc. Three methods of noninvasive evaluation of female, age and skin texture roughness, the relationship between elasticity [J]. Journal of sichuan university, medical sciences, 2009, 40 (2) : 364-366. 
[3]. ZHANG Mi wu X-iao ming CHU Xia, ChuXia.The Design of Cutis Health Management System Based On Images [J]. Microcomputer information, 2010 (2) : 178-180.

[4]. Peng-xiang xu. The research on evaluation system of skin quality[D]. Beijing university of technology, 2007.

[5]. GuChunJing da-lin jiang. The study of the skin texture evaluation method of the image processing [J]. Journal of instruments and meters, 2008 (z2) : 404-406.

[6]. Zhang Y T, Shen L N, Wu Z H, et al. Evaluation of Skin Viability Effect on Ethosome and Liposome - Mediated Psoralen Delivery via Cell Uptake[J]. Journal of pharmaceutical sciences, 2014, 103(10): 3120-3126.

[7]. Ms.tang, red-hatted. Noninvasive evaluation and applications in dermatology skin elasticity [J]. China cosmetic medicine, 2007, 12:710-712.

[8]. Gao yi. The BP neural network classifier optimization technology research. Ph.D. Dissertation of huazhong university of science and technology, 2012. 